\title{
Comparing the Diagnostic Performance of 2 Clinical Decision Rules to Rule Out Deep Vein Thrombosis in Primary Care Patients
}

Eit Frits van der Velde, $M D^{1}$

Diane B. Toll, $P b D^{2}$

Arina J. ten Cate-Hoek, MD PbD ${ }^{3}$

Ruud Oudega, $M D, P b D^{2}$

Henri E. J. H. Stoffers, MD, $P b D^{3}$

Patrick M. Bossuyt, MD, $P b D^{1}$

Harry R. Büller, $M D P b D^{1}$

Martin H. Prins, MD PbD

Arno W. Hoes, MD PbD

Karel G. M. Moons, MD, PbD ${ }^{2}$

Henk C. P. M. van Weert, MD PbD

${ }^{1}$ Academic Medical Center, Departments of General Practice, Vascular Medicine and Clinical Epidemiology, University of Amsterdam, The Netherlands

${ }^{2}$ University Medical Center Utrecht, Julius Center for Health Sciences and Primary Care, Utrecht, The Netherlands

${ }^{3}$ Maastricht Medical Centre, School of Public Health and Primary Care (CAPHRI), Departments of Epidemiology, General Practice, and Internal Medicine, Maastricht, The Netherlands

Conflicts of interest: authors report none.

\section{CORRESPONDING AUTHOR}

Eit Frits van der Velde, MD

Department of General Practice

Academic Medical Center

University of Amsterdam

Meibergdreef 15

1105 AZ Amsterdam, The Netherlands

e.f.vandervelde@amc.uva.nl

\begin{abstract}
PURPOSE The Wells rule is widely used for clinical assessment of patients with suspected deep vein thrombosis (DVT), especially in the secondary care setting. Recently a new clinical decision rule for primary care patients (the primary care rule) has been proposed, because the Wells rule is not sufficient to rule out DVT in this setting. The objective was to compare the ability of both rules to safely rule out DVT and to efficiently reduce the number of referrals for leg ultrasound investigation that would result in a negative finding.
\end{abstract}

METHODS Family physicians collected data on 1,086 patients to calculate the scores for both decision rules before leg ultrasonography was performed. In all patients D-dimer (dimerized plasmin fragment D) testing was performed using a rapid point-of-care assay. Patients were stratified into risk categories defined by each rule and the D-dimer result. Outcomes were DVT (diagnosed by ultrasonography) and venous thromboembolic complications or death caused by a possible thromboembolic event during a 90-day follow-up period. We calculated the differences between the 2 rules in the number of missed diagnoses and the proportions of patients that needed ultrasound testing.

RESULTS Data from 1,002 eligible patients were used for this analysis. A venous thromboembolic event occurred during follow-up in 7 patients with a low score and negative D-dimer finding, both with the Wells rule (7 of $447 ; 1.6 \%$; $95 \%$ confidence interval $[\mathrm{Cl}], 0.7 \%-3.3 \%)$ and the primary care rule (7 of $495 ; 1.4 \%$; $95 \% \mathrm{Cl}, 0.6 \%-3.0 \%)$. Using the Wells rule, 447 patients (45\%) would not need referral for further testing compared with 495 patients (49\%) when using the primary care rule (McNemar $P<.001$ ).

CONCLUSIONS In primary care, suspected DVT can safely be ruled out using either of the 2 rules in combination with a point-of-care D-dimer test. Both rules can reduce unnecessary referrals for compression ultrasonography by about $50 \%$, though the primary care rule reduces it slightly more.

Ann Fam Med 2011;9:31-36. doi:10.1370/afm.1198.

\section{INTRODUCTION}

W

ith an annual incidence of 1 to 2 per 1,000 inhabitants, deep vein thrombosis (DVT) is relatively rare in primary care, but it is feared for its associated morbidity and mortality if left untreated. ${ }^{1-4}$ When a family physician suspects DVT, most patients will be referred for additional testing. After the introduction of compression ultrasonography, the number of primary care patients referred for this noninvasive technique, which is highly accurate, increased progressively, partially because of improved accessibility and greater awareness for possible DVT. The result has been an increased number of diagnosed DVTs but at lessefficient use of resources and increased costs. ${ }^{5}$

Despite its widespread accessibility, ultrasonography may not be needed in all patients suspected of DVT. The availability of D-dimer (dimerized 
plasmin fragment D) testing made it possible to combine clinical assessment with this laboratory test to rule out DVT without the need for imaging tests. ${ }^{6} \mathrm{~A}$ diagnostic algorithm, based on a decision rule developed by Wells and colleagues that included information from a patient's medical history and physical examination, followed by D-dimer testing, is now used to guide management in many hospitals worldwide (Table 1). ${ }^{7}$ In the hospital setting the combination of a low clinical probability based on this decision rule and a negative D-dimer test result safely rules out DVT without the need for additional investigations. ${ }^{7,8}$ In all other cases, (serial) compression ultrasonography is indicated.

Concerns have been raised that this strategy may not be as safe in primary care. A validation study showed that the Wells rule did not adequately rule out DVT in primary care patients, as $2.9 \%$ (95\% confidence interval [CI] 2.5\%-3.3\%) of patients with DVT were missed, even after applying a qualitative, highly sensitive D-dimer test. ${ }^{9}$ This outcome was most likely because patient characteristics differed between primary and secondary care populations. One element in the Wells rule is an estimated probability of an alternative diagnosis, which primary care physicians and specialists might judge differently. The prevalence of thrombosis is low in primary care, and primary care physicians have a limited experience with it.

As a result, a specific primary care rule that included input from the patient history, physical examination, and a D-dimer test result (but without the subjective priorprobability estimation) was developed from data collected from 1,295 primary care patients with suspected DVT and referred for evaluation by ultrasonography. Of 16 variables, a model with 8 variables, including a quantitative D-dimer test result, was constructed using multivariate logistic regression analysis and excluding variables with a $P$ value of $>.10$ based on the log likelihood ratio test. For the decision rule, named the primary care rule, the regression coefficients of the variables were transformed to integers according to their relative contributions to the risk estimation (Table 1).5,10 The rule has been validated in various subgroups as well. ${ }^{11,12}$

A simple, rapid, bedside D-dimer test, using point-of-care assays performed with capillary blood, makes it possible to further enhance stratification of patients in the primary care setting. ${ }^{13}$ These qualitative D-dimer tests have a lower sensitivity and higher specificity than do quantitative tests. ${ }^{14}$

A diagnostic management study recently showed that primary care physicians can safely rule out DVT in approximately one-half of patients who they suspect have DVT by using the primary care rule (Table 1 ) and including a qualitative point-of-care D-dimer test. ${ }^{15}$ A direct comparison of the Wells rule with the primary care rule combined with a point-of-care D-dimer test in an unselected primary care population has never been performed. ${ }^{16}$ We therefore compared the safety and efficiency of both rules to rule out a diagnosis of DVT in primary care patients using the data from the above-mentioned study ${ }^{15}$ to determine which of these rules performs best. Because the Wells rule was derived and validated without D-dimer testing, and the primary care rule was derived and validated with quantitative D-dimer testing, we assessed both rules without D-dimer testing and then with a point-of-care qualitative D-dimer test.

\section{METHODS}

\section{Study Population}

The study included 1,086 unselected patients with clinically suspected DVT seen by more than 300 general practitioners in the Netherlands. The study's objective was to establish the safety and efficiency of ruling out DVT in primary care patients in a true management setting. The main results have been published elsewhere. ${ }^{15}$ Patients were eligible if they had 1 or more of the following symptoms: swelling, redness, or pain of the lower extremity. Patients were excluded if they were younger than 18 years or had received lowmolecular-weight heparin or vitamin $\mathrm{K}$ antagonists. The study was approved by the local review boards.

\section{Data Collection}

During the patient encounter, the primary care physicians registered all data needed to calculate the score
Table 1. Wells Rule and the Primary Care Rule Scoring to Rule Out Deep Vein Thrombosis (DVT)

\begin{tabular}{lcc}
\hline Variables & $\begin{array}{c}\text { Wells } \\
\text { Rule }\end{array}$ & $\begin{array}{c}\text { Primary } \\
\text { Care Rule }\end{array}$ \\
\hline Male sex & - & 1 \\
Oral contraceptive use & - & 1 \\
Presence of active malignancy (within last 6 mo) & 1 & 1 \\
Immobilization paresis/plaster lower extremities & 1 & - \\
Major surgery (last 3 mo) & 1 & 1 \\
Absence of leg trauma & - & 1 \\
Localized tenderness of deep venous system & 1 & - \\
Dilated collateral veins (not varicose) & 1 & 1 \\
Swelling, whole leg & 1 & - \\
Calf swelling $\geq 3$ cm & 1 & 2 \\
Pitting edema confined to the symptomatic leg & 1 & - \\
Previously documented DVT & 1 & - \\
Alternative diagnosis at least as likely as DVT & -2 & 6 \\
Positive D-dimer result & - & $\leq 3$ \\
Cutoff scores for considering DVT as absent & $\leq 1$ & - \\
\hline
\end{tabular}


of both decision rules, their most likely diagnosis, and the results of a D-dimer test on specific case record forms. All D-dimer testing was performed using a rapid point-of-care D-dimer assay (Clearview Simplify D-dimer Assay, Inverness Medical, Bedford, United Kingdom). Patient management was based on the primary care rule as calculated by the attending physician. Patients with scores of 3 or less were not referred for ultrasonography and were not prescribed anticoagulant treatment, whereas patients with scores of 4 or more were referred for compression ultrasonography. The case record forms were forwarded to the investigators immediately. Patients were followed up after 5 to 9 days by their physician.

Three months after entering the study, all patients received a questionnaire addressing signs and symptoms of venous thromboembolism. Patients who did not respond (30\%) were contacted through their general practitioners. Additional medical information was obtained about the patients from their general practitioners if any venous thromboembolic event was suspected. During 90 days of follow-up, venous thromboembolic complications and death caused by a possible thromboembolic event, confirmed by an independent adjudication committee, were recorded for all patients.

\section{Calculation of Both Scores}

To compare the safety and efficiency of the 2 rules, we calculated each patient's score on the Wells rule and recalculated the score on the primary care rule. The last item of the Wells rule (an alternative diagnosis as likely or more likely than DVT) was not explicitly coded by the primary care physicians. For this analysis, an alternate diagnosis was coded when the physician registered an alternative diagnosis from a prespecified list of 9 possible diagnoses.

For the Wells rule, patients with a score of 1 or less and a negative D-dimer result were assigned to the lowrisk group, and patients with a score of 2 or more or a positive D-dimer result were assigned to the high-risk group. For the primary care rule, a score of 3 or less and a negative $\mathrm{D}$-dimer result indicated a low risk, and a score of 4 or more or a positive D-dimer result, a high risk. Originally a D-dimer test result was incorporated in the primary care rule (Table 1$){ }_{1}^{5}$ whereas in the Wells rule it was taken into account after the clinical score was estimated. To be able to make a direct comparison between both rules, we computed the score of the primary care rule with and without the D-dimer test result.

\section{Missing Data}

A total of $2.7 \%$ of the patients had a missing values for 1 or more of the items included in the 2 rules. Because deleting records with missing values not only leads to reduced power but also to biased results, ${ }_{1}^{17-19}$ missing values were handled with multiple imputation, using the SAS procedure MI (SAS/STAT 9.2, SAS Institute, Cary, North Carolina).

\section{Outcome}

We assessed the safety of the Wells rule and the primary care rule by calculating the number of patients who during 3 months of follow-up had a missed diagnosis of a thromboembolic event defined as symptomatic venous thromboembolism, including fatal pulmonary embolism, nonfatal pulmonary embolism, and deep venous thrombosis. We also calculated the efficiency of both rules by comparing the number of patients that needed referral for ultrasonography.

\section{RESULTS}

Of the 1,086 patients, 58 met 1 or more of the exclusion criteria, and in another 26 patients, management was not completed according to protocol (eg, management without a decision rule and unsuccessful or other than point-of-care D-dimer testing). ${ }^{15}$

The data from the remaining 1,002 patients (97\%) were used for our analysis (Figure 1). The mean age of included patients was 58 years, and $37 \%$ were male. Suspicion of deep venous thrombosis was based on complaints of leg pain (87\%) and leg swelling (78\%). The median duration of symptoms was 5 days. Of 1,002 patients, 136 (14\%) had DVT confirmed by objective testing. Three patients were lost to follow-up.

Without including the D-dimer assay result, 607 patients $(61 \%)$ received scores indicating low risk of suspected DVT in both rules, and 130 (13\%) received scores indicating high-risk (Table 2). In 265 patients (26\%) the rules were discordant: the Wells score was high but the primary care score low in 243 patients $(24 \%)$, whereas in 22 patients (2\%) the primary care score was high but the Wells score was low.

Without additional D-dimer testing, by applying the Wells rule, 373 patients (37\%) were at high risk for DVT (score $\geq 1$ ), whereas by applying the clinical items of the primary care rule, 152 (15\%) were at high risk (score 24) These patients must be referred for ultrasonography regardless of D-dimer test results. In the patients not assessed as being at high risk for DVT, the D-dimer test was negative in 447 of 629 (71\%) for the Wells rule and in 495 of $850(58 \%)$ for the primary care rule. These patients would not need a diagnostic ultrasonography. This difference was significant (McNemar test; $P<.001$ ).

For both rules we found that 7 thromboembolic events occurred within the 3 months of follow-up in patients with a low score on the clinical items and a negative D-dimer: 7 of 447 (1.6\%) for the Wells rule 


\section{Figure 1. Study flow chart}

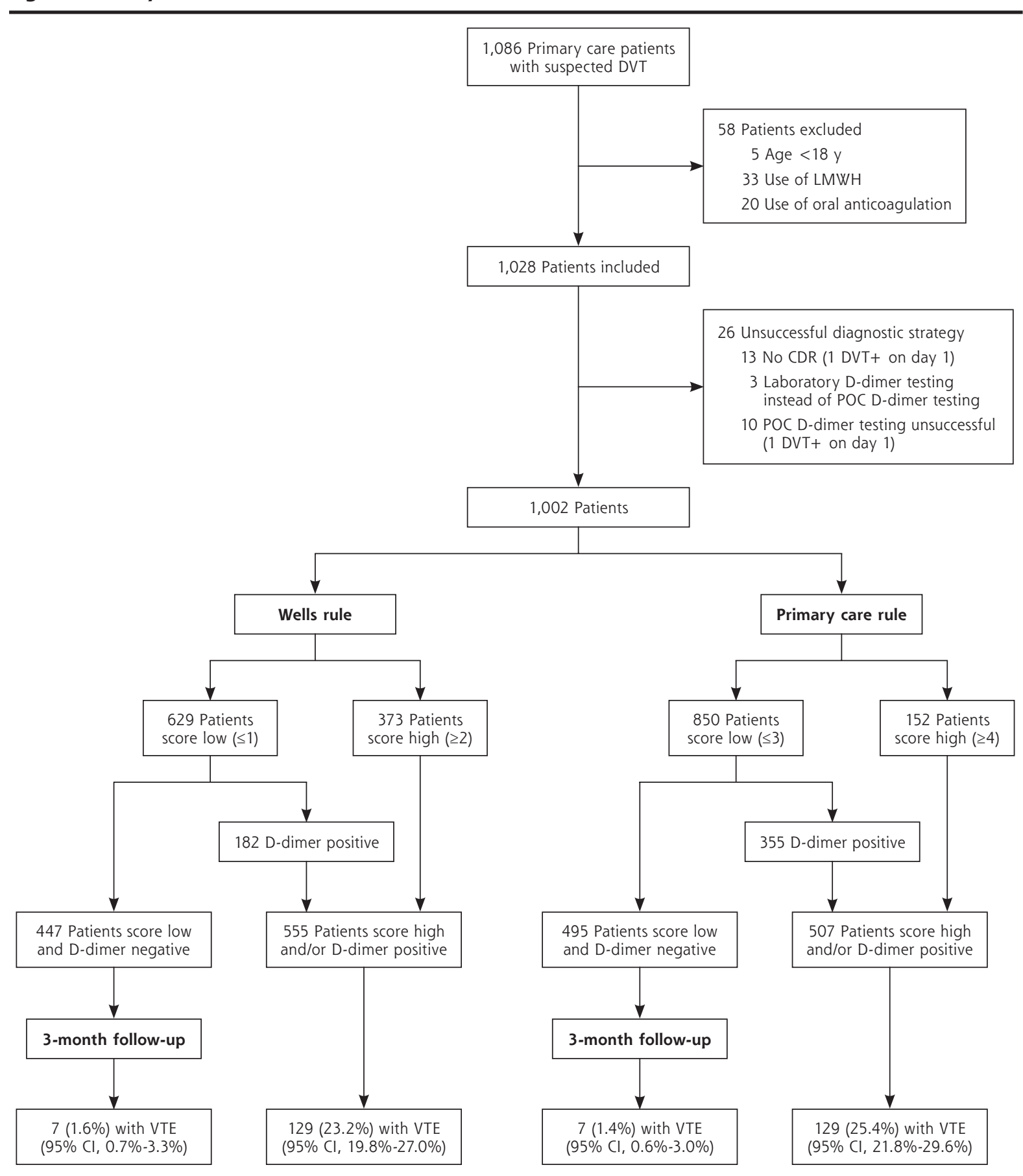

$\mathrm{Cl}=$ confidence interval; $\mathrm{CDR}=$ clinical decision rule; DVT = deep vein thrombosis; DVT+ = deep vein thrombosis confirmed by ultrasonography; $\mathrm{LMWH}=$ low-molecular-weight heparin; $\mathrm{POC}=$ point of care; $\mathrm{VTE}=$ venous thromboembolism.

and 7 of $495(1.4 \%)$ for the primary care rule (Table 3 ). Of these 7 patients, 4 would be missed by both rules, whereas in 3 patients the results of the decision rules were discordant.

When applying the decision rules and D-dimer testing, 555 patients $(55 \%)$ would be referred by the
Wells rule compared with 507 (51\%) by the primary care rule $(\mathrm{McNemar}$ test; $\mathrm{P}<.001)$. Of the 555 patients who would be referred for ultrasonography by the Wells rule, 129 (23\%) had DVT confirmed compared with 129 of 507 (25\%) when applying the primary care rule, a nonsignificant difference. $(P=.40)$ 
Table 2. Concordance Between the Wells Rule and Primary Care Rule Without the D-Dimer Test

\begin{tabular}{lccc}
\hline & \multicolumn{2}{c}{ Wells Rule } & \\
\cline { 2 - 3 } Primary Care Rule & $\begin{array}{c}\text { High Score, } \geq 2 \\
\text { No. (\%) }\end{array}$ & $\begin{array}{c}\text { Low Score, } \leq 1 \\
\text { No. (\%) }\end{array}$ & $\begin{array}{c}\text { Total } \\
\text { No. (\%) }\end{array}$ \\
\hline High score, $\geq 4$ & $130(13)$ & $22(2)$ & $152(15)$ \\
Low score, $\leq 3$ & $243(24)$ & $607(61)$ & $850(85)$ \\
Total & $373(37)$ & $629(63)$ & $1,002(100)$ \\
\hline
\end{tabular}

\section{Table 3. Patients With Low Scores on Both Rules, With and Without a Negative D-Dimer Result, and (Missed) Thromboembolic Events}

\begin{tabular}{|c|c|c|c|c|}
\hline \multirow[b]{2}{*}{ Outcome } & \multicolumn{2}{|c|}{$\begin{array}{l}\text { Wells Rule } \\
(N=1,002)\end{array}$} & \multicolumn{2}{|c|}{$\begin{array}{c}\text { Primary Care Rule } \\
\quad(N=1,002)\end{array}$} \\
\hline & No. (\%) & $95 \% \mathrm{Cl}$ & No. (\%) & $95 \% \mathrm{Cl}$ \\
\hline $\begin{array}{l}\text { Low CDR score without } \\
\text { D-dimer }\end{array}$ & $629(63)$ & $60-66$ & $850(85)$ & $83-87$ \\
\hline $\begin{array}{l}\text { Low CDR score and } \\
\text { D-dimer negative }\end{array}$ & $447(45)$ & $42-48$ & $495(49)$ & $47-54$ \\
\hline $\begin{array}{l}\text { VTE in patients with } \\
\text { low score and } \\
\text { D-dimer negative }\end{array}$ & $7(1.6)$ & $0.4-2.7$ & $7(1.4)$ & $0.6-2.9$ \\
\hline
\end{tabular}

could not ascertain that every consecutive patient was included, it is likely that our cohort is representative for all patients seen in primary care. Furthermore, we applied blinded assessment of outcomes by using ultrasonography or an independent adjudication committee that was not aware of the diagnostic procedure.

To appreciate our results, however, a few aspects need to be addressed. First, the items for both rules were recorded simultaneously, and we calculated the scores for both rules centrally and independently. Although the case record form was designed in such a way that clinical data were collected before D-dimer testing was performed, we cannot exclude that attending physicians documented the "presence of an alternative diagnosis," one of the most important items of the Wells rule, knowing the result of the primary care rule and of the D-dimer test. Usually such bias leads to a dilution of the difference between both tests, ie, the accuracy of the Wells rule and primary care rule become more alike. ${ }^{21-23}$ The D-dimer test result, however, will also be available in everyday practice, and given that the positive predictive value of the primary care rule for the clinical items is only $36 \%{ }^{15}$ and the negative predictive value is $91.7 \%$ (compared with $87.5 \%$ in the complete study population), the influence of this possible bias must not be exaggerated.

Second, the primary care rule was developed and validated as a comprehensive instrument that included clinical items and a quantitative D-dimer test. We compared the original Wells rule with the primary care rule without the D-dimer test by leaving out the D-dimer assay from the originally developed rule. As a result, the relative weights of the remaining items would likely be higher because part of the information covered by the deleted D-dimer test would be included by the other items. We did not adjust for this likelihood, possibly underestimating the performance of the primary care rule without the D-dimer assay compared with the Wells rule.

Finally, judged against previous studies carried out with quantitative tests, the Wells rule and point-of-care D-dimer testing performed better than expected ${ }^{9,11}$ in that our results were more similar to those of earlier studies in secondary care. ${ }^{24}$ Our findings could be explained by a different performance of the point-ofcare D-dimer test. Other potential explanations might be the direct comparison of both rules in this study and a different case mix. We included all patients suspected of having a DVT by their primary care physi- 
cian, whereas in most previous studies patients were included when a primary care physician judged ultrasound testing to be necessary. ${ }^{24}$

The overall prevalence of $14 \%$ confirmed venous thromboembolic events strengthens the external validity of our findings. This prevalence is somewhat lower than found in earlier studies, probably because we performed a primary care-based study. D-dimer testing was performed with capillary whole blood using a point-of-care assay. In this way D-dimer measurement could be performed in the general practitioner's office or at the patient's home. The D-dimer test alone in this setting had a sensitivity of $86 \%$ and a specificity of $61 \% .{ }^{15}$ The relatively high specificity contributed to a good clinical efficiency, ie, a relatively large proportion of patients could safely be spared referral for ultrasonography. Using another (less-specific) assay, as was the case in previous studies that used a laboratory based D-dimer test with venous blood, might result in more referrals for imaging. ${ }^{25}$

This study shows that DVT can safely be ruled out in primary care by using either decision rule. A low clinical probability, in combination with a negative point-of-care D-dimer test result, spares almost 50\% of the patients traveling to a secondary care facility for ultrasonography.

When using the primary care rule, in every 100 patients at least 4 ultrasound procedures can be prevented at the cost of 22 additional D-dimer assays ( 5 to 6 assays per ultrasound test spared). Direct medical costs per patient thus will be about the same, but when using the primary care rule, fewer patients will be referred for ultrasonography. The relatively compact primary care rule, which does not require a subjective prior-probability estimation, therefore seems more convenient for both patients and for physicians.

To read or post commentaries in response to this article, see it online at http://www.annfammed.org/cgi/content/full/9/1/31.

Submitted October 23, 2009; submitted, revised, July 31, 2010; accepted, August 27, 2010.

Key words: Venous thrombosis; venous thromboembolism; D-dimer; clinical decision support systems; primary health care

Funding support: This study was supported by The Netherlands Organization for Scientific Research (ZON-MW), grant No. 945-04-009.

\section{References}

1. Huerta C, Johansson S, Wallander MA, García Rodríguez LA. Risk factors and short-term mortality of venous thromboembolism diagnosed in the primary care setting in the United Kingdom. Arch Intern Med. 2007;167(9):935-943.

2. Kilroy DA, Ireland S, Reid P, Goodacre S, Morris F. Emergency department investigation of deep vein thrombosis. Emerg Med J. $2003 ; 20(1): 29-32$
3. Prandoni P, Lensing AW, Cogo A, et al. The long-term clinical course of acute deep venous thrombosis. Ann Intern Med. 1996;125(1):1-7.

4. White RH. The epidemiology of venous thromboembolism. Circulation. 2003;107(23)(Suppl 1):14-18.

5. Oudega R, Moons KG, Hoes AW. Ruling out deep venous thrombosis in primary care. A simple diagnostic algorithm including D-dimer testing. Thromb Haemost. 2005;94(1):200-205.

6. Kraaijenhagen RA, Piovella F, Bernardi $E$, et al. Simplification of the diagnostic management of suspected deep vein thrombosis. Arch Intern Med. 2002;162(8):907-911.

7. Wells PS, Owen C, Doucette S, Fergusson D, Tran H. Does this patient have deep vein thrombosis? JAMA. 2006;295(2):199-207.

8. Ten Cate-Hoek AJ, Prins MH. Management studies using a combination of D-dimer test result and clinical probability to rule out venous thromboembolism: a systematic review. J Thromb Haemost. 2005;3(11):2465-2470.

9. Oudega R, Hoes AW, Moons KG. The Wells rule does not adequately rule out deep venous thrombosis in primary care patients. Ann Intern Med. 2005;143(2):100-107.

10. Oudega R, Hoes AW, Toll DB, Moons KG. The value of clinical findings and $\mathrm{D}$-dimer tests in diagnosing deep vein thrombosis in primary care. Semin Thromb Hemost. 2006;32(7):673-677.

11. Toll DB, Oudega R, Bulten RJ, Hoes AW, Moons KG. Excluding deep vein thrombosis safely in primary care. J Fam Pract. 2006;55(7): 613-618.

12. Toll DB, Oudega R, Vergouwe Y, Moons KG, Hoes AW. A new diagnostic rule for deep vein thrombosis: safety and efficiency in clinically relevant subgroups. Fam Pract. 2008;25(1):3-8.

13. Van Der Velde EF, Wichers IM, Toll DB, Van Weert HC, Büller HR. Feasibility and accuracy of a rapid 'point-of-care' D-dimer test performed with a capillary blood sample. J Thromb Haemost. 2007;5(6): 1327-1330.

14. Geersing GJ, Janssen KJ, Oudega R, et al. Excluding venous thromboembolism using point of care D-dimer tests in outpatients: a diagnostic meta-analysis. BMJ. 2009;339:b2990.

15. Büller HR, Ten Cate-Hoek AJ, Hoes AW, et al; AMUSE (Amsterdam Maastricht Utrecht Study on thromboEmbolism) Investigators. Safely ruling out deep venous thrombosis in primary care. Ann Intern Med. 2009;150(4):229-235.

16. Reilly BM, Evans AT. Much ado about (doing) nothing. Ann Intern Med. 2009;150(4):270-271.

17. Gornbein JA, Lazaro CG, Little RJ. Incomplete data in repeated measures analysis. Stat Methods Med Res. 1992;1(3):275-295.

18. Greenland S, Finkle WD. A critical look at methods for handling missing covariates in epidemiologic regression analyses. Am J Epidemiol. 1995;142(12):1255-1264

19. Donders AR, van der Heijden GJ, Stijnen T, Moons KG. Review: a gentle introduction to imputation of missing values. J Clin Epidemiol. 2006;59(10):1087-1091

20. Goodacre S, Sampson F, Thomas S, van Beek E, Sutton A. Systematic review and meta-analysis of the diagnostic accuracy of ultrasonography for deep vein thrombosis. BMC Med Imaging. 2005;5:6.

21. Begg CB, McNeil BJ. Assessment of radiologic tests: control of bias and other design considerations. Radiology. 1988;167(2):565-569.

22. Swets JA. Measuring the accuracy of diagnostic systems. Science. 1988;240(4857):1285-1293.

23. Moons KG, Grobbee DE. When should we remain blind and when should our eyes remain open in diagnostic studies? J Clin Epidemiol. 2002;55(7):633-636.

24. Goodacre S, Sutton AJ, Sampson FC. Meta-analysis: The value of clinical assessment in the diagnosis of deep venous thrombosis. Ann Intern Med. 2005;143(2):129-139.

25. Goodacre S, Sampson FC, Sutton AJ, Mason S, Morris F. Variation in the diagnostic performance of D-dimer for suspected deep vein thrombosis. QJM. 2005;98(7):513-527. 